\title{
Ein Maulbronner Fragment der lateinischen Talmudübertragung des 13. Jahrhunderts (mit Edition)*
}

\author{
Von Görge K. Hasselhoff und Óscar de la Cruz
}

In seiner Torso gebliebenen Doktorarbeit stellte Anfang der 1980er Jahre Eberhard Goнl die Frage nach einer Buchbinderei im Skriptorium des Klosters Maulbronn. Er kam dabei zu dem Schluss: „Auszuschließen ist die Existenz einer solchen Werkstatt nicht, und die Einrichtung einer Klosterbuchbinderei in den siebziger Jahren des fünfzehnten Jahrhunderts würde wohl mit dem zusammenpassen, was man sonst über die Entwicklung des Maulbronner Buchwesens in dieser Epoche weiß." ${ }^{\text {E1 }}$ Ein kürzlich entdecktes Talmudfragment aus Maulbronn (Abb. 1, 2) gibt nun einen neuen Anhalt für die Existenz einer solchen Buchbindewerkstatt.

Bei Arbeiten am Dachstuhl im Klosterhof fand sich im August 2013 im Bereich der Dachtraufe über dem sogenannten Faustloch, einem kleinen eingewölbten Raum, der sich westlich der Bibliothek im 1. Obergeschoss des Nordquerhauses der Klosterkirche und oberhalb der südöstlichen Kreuzgangecke befindet, ein auf der Innenseite mit einem Pergament geschützter Holzdeckel vom Ende des 15. Jahrhunderts eines ansonsten verschollenen Buchs, das laut Deckelinschrift den Titel „Liber miraculorum ordinis cisterciensis“ trug². Diese Aufschrift und der Fundort lassen es nicht unwahrscheinlich erscheinen, dass der Kodex tatsächlich in der angenommenen Buchbinderwerkstatt hergestellt wurde. Das als Makulatur

Die nachfolgende Untersuchung wurde angeregt durch Andreas Trau b (Tübingen) und Peter Rǘckert (Stuttgart), die um eine Expertise des Fragments baten. Zugleich ist sie Teil des ERC-Projekts „The Latin Talmud and Its Influence on Christian-Jewish Polemic“ (European Union's Seventh Framework Programme (FP7/2007-2013) / ERC Grant agreement $\mathrm{n}^{\circ}$ 613694); für zahlreiche Kommentare und Hinweise danken wir unserem Kollegen Alexander Fidora (Bellaterra, Barcelona). - Für den ersten Teil des Beitrags zeichnet G. K. Hasselhoff verantwortlich, für die Edition beide Autoren.

${ }_{1}^{1}$ Eberhard GoHL, Studien und Texte zur Geistesgeschichte der Zisterzienserabtei Maulbronn im späten Mittelalter, Ms.-Diss. phil. Tübingen 1980, S. 150.

${ }^{2}$ Vgl. Staatliche Schlösser und Gärten Baden-Württemberg, Presseinformation vom 15. April 2014 sowie Emails von Holger Рвовsт (Maulbronn) vom 16. und 17. April 2014 (dort auch die Fundortbeschreibung). 
verwendete Pergament (Ende 13. Jahrhundert ${ }^{3}$ ) dagegen scheint aus Frankreich, möglicherweise aus Paris zu stammen; wie es nach Maulbronn gekommen ist, lässt sich nicht mehr rekonstruieren.

Stellt schon der Fund an sich eine Besonderheit dar - auch wenn der Deckel ausweislich der in der Umgebung eingelagerten Texte aus dem Anfang des 20. Jahrhunderts erst spät an seinen Fundort gelangt war ${ }^{4}$ - so gilt das erst recht für das verarbeitete Pergamentblatt, das seinerseits ein Teil einer Abschrift einer lateinischen Talmudübertragung aus dem 13. Jahrhundert ist.

\section{Die lateinische Talmudübertragung aus der Mitte des 13. Jahrhunderts ${ }^{5}$}

Der Talmud als verschriftlichte mündliche Tora enthält zwar überwiegend alte Traditionen, ist als schriftliches Werk jedoch eher jüngeren Datums. Wie kürzlich Talya Fishman in einer exzellenten Überblicksstudie zusammengefasst hat, gelangten schriftliche Fassungen einzelner Talmudtraktate und schließlich des gesamten Babylonischen Talmuds erst ungefähr im 11. Jahrhundert nach Mitteleuro$\mathrm{pa}^{6}$. Ebenso finden sich auch erst ab dem 12. Jahrhundert erste Spuren talmudischer Schriften in christlicher Literatur, noch eher allgemein bei Petrus Alfonsi ${ }^{7}$ und dann mit dem Begriff „Talmud“ bei Petrus Venerabilis ${ }^{8}$. Es bleibt jedoch bei Einzelvorkommen. Das ändert sich im 13. Jahrhundert.

Gegen Ende der 1230er Jahre trat der aus dem Judentum zum Christentum konvertierte Nicolaus Donin mit einer Anklage gegen den Talmud auf, in deren Gefolge der Talmud als eigene jüdische Literaturgattung einem breiteren christlichen Publikum bekannt wurde. Nikolaus Donin hat 1239 zunächst bei seinem Ortsbischof Odo und dann bei Papst Gregor IX. versucht, den Talmud verbieten zu lassen, weil sich in diesem Aussagen gegen das Christentum fänden und er auch an-

${ }^{3}$ Jesús Alturo i Perucho (Bellaterra, Barcelona) wies darauf hin, dass die Abschrift möglicherweise eher Anfang des 14. Jahrhunderts anzusetzen sei.

${ }^{4}$ Hinweis von Holger РвовSт in der Email vom 17. April 2014.

${ }^{5}$ In den nachfolgenden Abschnitt sind Teile eines Vortrags beim Interdisziplinären Symposium „Vergessene Texte des Mittelalters“, Göttingen, 2.-4. Dezember 2011, eingeflossen.

${ }^{6}$ Vgl. Talya Fishmann, Becoming the People of the Talmud. Oral Torah as Written Tradition in Medieval Jewish Cultures, Philadelphia 2011.

7 Vgl. zusammenfassend Görge K. Hasselhoff, Petrus Alfonsis Judentum vor dem Hintergrund seiner Zeit, in: Carmen Cardelle de Hartmann/Philipp Roelli (Hg.), Petrus Alfonsi und die Religionspolemik. Hintergrund, Kontexte und Rezeption des Dialogus Petrus Alfonsi and Religious Polemics. Backgrounds, Contexts and Reception of his Dialogus, Florenz 2015, S. 61-76 (im Druck).

${ }^{8}$ Vgl. Petrus Venerabilis, Adversus Iudeorum inveteratam duritiem, hg. von Yvonne Friedman (Corpus Christianorum. Continuatio Mediaevalia, Bd.58), Turnhout 1985, Tit. V, lin. 1087-1105 (S. 156f.); lin. 1336-1430 (S. 163-166). 
sonsten ein blasphemisches Werk sei ${ }^{9}$. In der Folge berief der Bischof eine öffentliche Disputation ein, in der Nikolaus Donin mit Yechiel von Paris über die Irrtümer des Talmud disputieren sollte. Diese Disputation hat 1240 in Paris stattgefunden. Für die Anklage selbst übersetzte Nicolaus einige wenige Auszüge aus dem Talmud.

In der Folge, 1241, 1242 oder 1244, kam es zur Verbrennung des Talmud in Paris $^{10}$. Angeblich sollen 24 Wagenladungen voll Talmudexemplaren verbrannt worden sein; allerdings hat Colette Sirat nachgewiesen, dass diese Zahl bei weitem übertrieben ist ${ }^{11}$. Dennoch ist festzuhalten, dass jüdische Schriften - wahrscheinlich nicht nur der Talmud, sondern auch Gebetbücher oder anderes - verbrannt wurden, auch wenn die wirkliche Zahl unklar bleibt. In der Folge gibt es Versuche - die dann später auf der Iberischen Halbinsel noch viel wirksamer werden -, dass Talmudexemplare zensiert und alles das, was mutmaßlich anti-christlich war, getilgt wurde.

Nikolaus Donin war mit dem noch jungen Dominikanerorden assoziiert, der einen Konvent in Paris (Saint-Jacques) betrieb und dort durch den Übertritt zweier Magister der jungen Sorbonne, die ihre Lehrstühle mitgebracht hatten, schnell Fuß gefasst hatte. In diesem Dominikanerkonvent lebte zu der Zeit allem Anschein nach der Ordensmeister Ramon de Penyafort (ca. 1180-1275); Prior war Theobaldus von Saxonia, wahrscheinlich Thibaud von Sézanne (bezeugt 1248) ${ }^{12}$. Später kam Albertus Magnus (1200-1280) als Lehrer dazu, der uns hier am Rande interessiert. Unter der Leitung von Ramon de Penyafort hatte sich der Dominikanerorden zum Ziel gesetzt, die Missionsbestrebungen zu verstärken und endlich alle „un-

9 Vgl. A. Lewin, Die Religionsdisputation des R. Jechiel von Paris 1240 am Hofe Ludwigs, des Heiligen, ihre Veranlassung und ihre Folgen, in: Monatschrift für Geschichte und Wissenschaft des Judenthums 18 (1869) S. 97-110, S. 145-156, S.193-210; Isidore Loeb, La controverse de 1240 sur le Talmud, in: Revue des études juives 1 (1880) S. 247-261, 2 (1881) S. 248-270, 3 (1881) S. 39-57; Chen Merchavia, The Church Versus Talmudic And Midrashic Literature (500-1248), Jerusalem 1970 (Hebr.); Нуаm Массову (Hg.), Judaism on Trial. Jewish-Christian Disputations in the Middle Ages, London 1982 (mehrere Nachdrucke), S. 19-38, 153-167, 216, 218 f.; Gilbert Dahan (Hg.), Le brûlement du Talmud à Paris 12421244, Paris 1999; The Trial of the Talmud. Paris, 1240. Hebrew Texts translated by John Friedman. Latin Texts translated by Jean Connell Hoff. Historical Essays by Robert CHAZAN (Mediaeval Sources in Translation, Bd. 53), Toronto 2012.

10 Vgl. André Tuilier, La condemnation du Talmud par les maîtres universitaires parisiens. Ses causes et ses conséquences politiques et idéologiques, in: DAHAN (wie Anm. 9) S. 5978; Paul Lawrence Rose, When was the Talmud burnt at Paris? A critical examination of the Christian and Jewish sources and a new dating: June 1241, in: Journal of Jewish Studies 62 (2011) S. 324-339; Judah Galinsky, The Different Hebrew Versions of the "Talmud Trial' of 1240 in Paris, in: Elisheva Carlebach/Jacob J. Schacter (Hg.), New Perspectives on Jewish-Christian Relations (The Brill Reference Library of Judaism, Bd.33), Leiden/Boston 2012, S. 109-140.

${ }^{11}$ Colette Sirat, Les manuscrits du Talmud en France du Nord au XIII ${ }^{e}$ siècle, in: Dahan (wie Anm. 9) S.121-139, v.a. 127.

12 Gilbert Dahan, Les traductions latines de Thibaud de Sézanne, in: Ders. (wie Anm. 9) S. $95-120$. 
gläubigen“ Juden zum Christentum zu konvertieren, weswegen der Orden auch die Disputation unterstützt hat. Um den eigenen Anliegen ein breiteres Gewicht zu geben, scheint im Anschluss an Donins Vorarbeiten eine neue, sehr viel ausführlichere (Auswahl-) Übertragung angefertigt worden zu sein. Diese war nun in der Reihenfolge der dem Konvent vorliegenden Talmudausgabe angefertigt ${ }^{13}$, um die Gottlosigkeit und Blasphemien des Talmud aufzuzeigen ${ }^{14}$. Möglicherweise war der Prior Theobaldus von Saxonia in die Übersetzung involviert, zumindest gibt es noch eine Verarbeitung in dessen Pharetra ${ }^{15}$, wobei er möglicherweise auf seine eigene Übersetzung zurückgegriffen hat, um dann ausführlicher gegen den Talmud zu schreiben.

Einige Jahre später arrangierte ein anderer, ebenfalls anonym gebliebener Übersetzer die beiden ihm vorliegenden Übersetzungen neu, nun in systematischer Hinsicht nach Themen ${ }^{16}$. Die einzelnen Übertragungen sind sehr unterschiedlich überliefert.

Die Donin'sche thesenhafte Übertragung ist in einer mittelalterlichen Pariser Handschrift (Bibliothèque nationale de France, Ms. lat. 16.558) ${ }^{17}$ und einer frühneuzeitlichen Kopie derselben (Bibliothèque Mazarine, Ms. 1115) ${ }^{18}$ sowie in den beiden schon länger bekannten Handschriften aus Carpentras (Bibliothèque Inguimbertine, lat. 153) ${ }^{19}$ und Girona (Arxiu Capitular, Ms. 19b) ${ }^{20}$ überliefert. Hier fin-

13 Vgl. die Übersicht bei José María Millás Vallicrosa, Extractos del Talmud y alusiones polémicas en un manuscrito de la Biblioteca Catedral de Gerona, in: Sefarad 20 (1960) S. 17-49, hier S. 24f.; Merchavia (wie Anm. 9) S. 364-418.

${ }^{14}$ Vgl. die Auszüge aus dem Manuskript der Bibliothèque nationale de France, Paris, Ms. lat. 16.558 bei Erich Klibansky, Beziehungen des christlichen Mittelalters zum Judentum, in: Monatsschrift für Geschichte und Wissenschaft des Judentums 77 (1933) S. 456-462, hier S. 459-462.

15 Zu den Pharetra und der möglichen Autorschaft von Nikolaus von Straßburg vgl. Carmen Cardelle de Hartmann, Drei Schriften mit dem Titel „Pharetra fidei“, in: Aschkenas 11 (2001) S. 327-349; Dies., Lateinische Dialoge 1200-1400. Literaturhistorische Studie und Repertorium (Mittellateinische Studien und Texte, Bd. 37), Leiden u.a. 2007, S. 388-392.

16 Vgl. hierzu Alexander Fidora, The Latin Talmud and its Translators: Thibaud de Sézanne vs. Nicholas Donin?, demnächst in: Henoch. Historical and Textual Studies in Ancient and Medieval Judaism and Christianity. - Alexander PAтschovsкy, Der „Talmudjude“: Vom mittelalterlichen Ursprung eines neuzeitlichen Themas, in: Alfred Haverkamp/ Franz-Josef Ziwes (Hg.), Juden in der christlichen Umwelt des späten Mittelalters (Zeitschrift für historische Forschung. Beiheft, Bd.13), Berlin 1992, S. 13-27, hier S. 18, gibt eine andere Reihenfolge; diese Reihung scheint jedoch unhaltbar zu sein.

17 Eine hilfreiche Inhaltsübersicht hat DAHAN (wie Anm.12).

18 Nach eigener Autopsie im März 2012; das Fragment umfasst f. 46r-136v, vgl. Valentin Rose, Verzeichniss der Lateinischen Handschriften der Königlichen Bibliothek zu Berlin, 2. Bd.: Die Handschriften der Kurfürstlichen Bibliothek und der Kurfürstlichen Lande, Erste Abteilung (Die Handschriften-Verzeichnisse der Königlichen Bibliothek zu Berlin, Bd. 13), Berlin 1901, S. 473-479, v.a. S. 475-479.

${ }^{19}$ Catalogue général des manuscrits des Bibliothèques Publique de France, Départments 34, Carpentras, par M. Duhamel, Tome I, Paris 1901, S. 85 f.

20 Vgl. die Beschreibung der Handschrift bei Millás Vallicrosa (wie Anm.13); zum 
det sie sich im Anschluss an die umfassendere Sammlung der „Extracciones ex Talmud“, die außer in den genannten noch in zwei weiteren Handschriften als Fragment überliefert ist: In einer Berliner Handschrift (Staatsbibliothek, Ms. theol. lat. fol. 306 $)^{21}$ sowie in einem von Joseph KLAPPER bereits 1926 edierten Einbandfragment aus Wrocław (Breslau) (Bibl. Uniw., MS I Q 134a) ${ }^{22}$ finden sich unterschiedlich umfangreiche Abschnitte dieser Übertragung. Der mutmaßlich ältesten, der Pariser Handschrift ist die systematische Kompilation mit Neuübersetzungen vorangestellt. Von dieser Kompilation waren bislang nur zwei Exemplare (zuzüglich einer unmittelbaren Kopie) bekannt: eben die Pariser Handschrift sowie eine Handschrift aus Schaffhausen (Ministerialbibliothek, Min. 71) ${ }^{23}$. Mit dem vorliegenden Maulbronner Einbandfragment ändert sich die Situation nun, weil wir einen dritten Textzeugen vorliegen haben, der belegt, dass auch diese dritte Übertragung weite(re) Verbreitung gefunden hatte.

Es gibt eine Besonderheit in allen drei Übertragungen, weil nicht nur Teile der Mischna und der Gemara übertragen sind, sondern auch „Glossen Salomos“, d.h. es sind Kommentare des wohl bedeutendsten aschkenasischen Kommentators Rashi (R. Salomon aus Troyes) mit eingefügt ${ }^{24}$. Für die benutzte Vorlage bedeutet das, dass es sich um ein aschkenasisches Manuskript gehandelt haben muss, das schon Raschi als Teil des Talmud kodifiziert hatte, und dem Übersetzer war zwar klar, dass die Kommentierung etwas Besonderes ist - deshalb ist sie abgesetzt als glosa solomonis -, aber gleichzeitig läuft diese Glosse mit als Teil des Talmud. In der Vorlage scheinen zudem die Namen der Rabbinen z.T. anders zu sein, als sie im heutigen Mehrheitstext zu finden $\operatorname{sind}^{25}$.

Was macht nun diese Übertragung aus? Zunächst einmal handelt es sich um eine extreme Auswahlübertragung. Studiert man den lateinischen Text für sich allei$n e^{26}$, vergeht einem schnell die Freude an der Lektüre: Es handelt sich um irritie-

Verhältnis zur bzw. der möglichen Nichtabhängigkeit von der Pariser Handschrift vgl. Alexander Fidora, Die Handschrift 19b des Arxiu Capitular de Girona: Ein Beitrag zur Überlieferungsgeschichte des lateinischen Talmud, demnächst in: FS Klaus Herbers.

${ }^{21}$ Nach eigener Autopsie im November 2012.

22 Das Fragment ist ediert in: Joseph KLAPper, Ein Florilegium Talmudicum des 13. Jahrhunderts, in: Literaturwissenschaftliches Jahrbuch der Görresgesellschaft 1 (1926) S.3-23.

${ }^{23}$ Vgl. Rudolf Gamber u.a., Die mittelalterlichen Handschriften der Ministerialbibliothek Schaffhausen, Dietikon/Zürich 1994, S. $176 \mathrm{f}$.

${ }^{24}$ Vgl. dazu G. K. Hasselhoff, Lateinische Übertragungen jüdischer religiöser Texte im 13. Jahrhundert als Beitrag zu einer Friedenskultur? - Eine Skizze, in: Arnulf von ScheLina/Eveline Goodman-Thau (Hg.), Zwischen Formation und Transformation. Die Religionen Europas auf dem Weg des Friedens, Göttingen 2011, S. 121-130, hier S. 122 f., 126.

${ }^{25}$ Vgl. z.B. M f. 1va Z.22: aus Shimlaj wird „Symeon“; Z.27: aus Jehuda wird „euehu“; Z. 32 aus Jehuda wird „huza“, usw. - Dass das keineswegs ungewöhnlich ist, hat Andreas LehnardT gezeigt, vgl. Ders., Die Kasseler Talmudfragmente (Schriftenreihe der Universitätsbibliothek Kassel - Landesbibliothek und Murhardsche Bibliothek der Stadt Kassel, Bd. 9), Kassel 2007.

${ }^{26}$ In gedruckter Form liegen bislang nur eine Transkription der Breslauer Fragmente bei

Zeitschrift für Württembergische Landesgeschichte 74 (2015), S. 331-344.

(C) Kommission für geschichtliche Landeskunde in Baden-Württemberg und Württembergischer

Geschichts- und Altertumsverein e.V.

ISSN 0044-3786 
rend kurze, mit zahlreichen Fremd- bzw. Lehnwörtern durchsetzte Sätze, deren Auswahl den Anschein von Verwirrtheit erweckt. Legt man allerdings daneben eine Talmudausgabe, dann stellt man schnell fest, dass Kerngedanken talmudischer Disputationen - oft extrem verkürzt - übertragen worden sind, aber sie sind für den lateinischen Leser wegen der Kürzungen eigentlich kaum brauchbar gewesen. Übertragen worden sind in unterschiedlichem Umfang Teile der Traktate Berakhot, Shabbat, Bava Qama, Bava Metsia, Bava Batra, Sanhedrin, Makkot, Shevuot, Avoda Zara sowie Summarien der Ordnungen (Seder) Moed, Nashim und Qodashim. Das heißt, aus fast allen Traktaten des Talmud sind Einzelsätze, Einzelbestimmungen oder ganze Passagen übersetzt worden. Wenn man sie genauer anschaut, sind die einzelnen Übertragungen jedoch - abgesehen davon, dass sie ziemlich aus dem Zusammenhang gerissen sind - sehr wörtlich übertragen. Bis dahin, dass Termini, die für ein lateinisches Publikum unverständlich waren, auch als solche stehengeblieben sind: z.B. „aspen“ für „arpad“, „kymos“ für „qimus“, „hooa“ für „choya“ usw. Das Problem bei diesen Termini ist, dass es auch bei den hebräischen Kommentatoren Diskussionen darüber gibt, wie man diese übertragen soll. Man weiß es nicht, und auch der Übersetzer hat es nicht gewusst und daher das Lehnwort stehenlassen.

Interessanter wird es dann in anderen Bereichen. Ich möchte ein Beispiel geben, das zeigt, dass diese Übertragung - wenn man Einzelsätze daraus genommen hat - durchaus auch eine Relevanz für ein christliches Zielpublikum haben konnte. Die Bedeutsamkeit besteht darin, dass eine Kenntnis vom Judentum vermittelt worden ist, die das Neue darstellte, welches das Judentum, das sich auf den Talmud stützte, erläutert. Beispielsweise werden aus dem Traktat Qiduschin Fol.29b-30a folgende zwei Sätze übersetzt: Dan docuit Zabulon filium suum per Mykra et Mysna et Talmuz et Halakoz et Agazon. glosas. ${ }^{27}$ Und dann weiter:

Semper debet homo tercionare annos suos, tercium in Mykra, tercium in Mysna et tercium in Talmuz. Scitne, quam diu vivet? Sed hoc dicitur de diebus. ${ }^{28}$

Der Kontext der Aussagen ist - unter Zuhilfenahme des talmudischen „Originals“ - leicht zu erschließen: Zebulon, Sohn des Dan wurde vom Großvater unterrichtet. Die unterrichtete Literatur umfasste fünf Bereiche: a) die Schrift (mykra, hebräisch: miqrab), b) die Mischna (mysna), also den „Kern“ des Talmud, c) den Talmud, wiedergegeben in einer Transkription in aschkenasischer Aussprache als „talmuz“, also die Gemara, die Auslegung der Mischna. Diese Gemara ist unterteilt in d) Halakoz, das sind die Hilkhot bzw. Halakhot, d.h. die Zusammenfas-

Klapper (wie Anm. 22), sowie Auszüge der Handschriften aus der Bibliothèque nationale de France in Paris und aus Girona vor bei Klibansky (wie Anm. 14), Millás Vallicrosa (wie Anm.13) und bei Dahan (wie Anm.12); eine Gesamtausgabe mitsamt umfassender Handschriftenbeschreibung und Stemmatisierung im Rahmen des in der ersten Anm.genannten Projekts ist jedoch in Arbeit.

27 Zu bQid 29b-30a, zit. nach Klapper (wie Anm. 22), S. 17, Z. 258 f.

28 Ebd., Z.265-267. 
[Nach S. 226 folgt eine Kunstdrucktafel, deren Abbildungen aus rechtlichen Gründen online nicht bereitgestellt werden können:]

Abb. 1: Talmud-Fragment, Kloster Maulbronn, Recto-Seite (Staatliche Schlösser und Gärten Baden-Württemberg, Foto: Magdalena Liedtke).

Abb. 2: Talmud-Fragment, Kloster Maulbronn,Verso-Seite (Staatliche Schlösser und Gärten Baden-Württemberg, Foto: Magdalena Liedtke). 
sung der einzelnen Bestimmungen der Halakha in Kapiteln oder Teilen, sowie e) die Agazon, das sind die Aggadot, also die erzählenden Teile bzw. lehrreichen Erzählungen. Durch die parataktische Anordnung et-et-et in der lateinischen Übersetzung ist jedoch zugleich die Schwierigkeit gegeben, dass der unvoreingenommene Leser von fünf Texten ausgehen muss, weil nicht erkennbar ist, dass die Sammlungen Miqra (mykra) und Mischna (mysna) von den Gattungen Halakha und Haggada zu unterscheiden sind. Die kundige Leserin oder der kundige Leser kann das jedoch im zitierten Fortgang dann erschließen: Dort heißt es, in drei Schritten solle man lernen: ein Drittel des Lebens mit der Schrift, ein Drittel des Lebens mit der Mischna und ein Drittel des Lebens mit dem Talmud. Allerdings solle man nicht in Lebenszeitabschnitten rechnen, sondern in Wochenabschnitten, und zwar von den sechs Tagen der Woche an je zwei Tagen Schrift, an zwei Tagen Mischna, an zwei Tagen Talmud (und am Shabbat sollst du ruhen).

Nicht alle Auszüge sind so lehrreich wie diese beiden, denn die Intention der Übersetzer war es, neben einer groben Kenntnis des Talmud, vor allem eine Kenntnis der Irrtümer und antichristlichen Polemiken des Werks aufzuzeigen und gleichzeitig zu zeigen, was für eine mindere literarische Qualität dem Talmud zukommt. Soviel zur (Haupt-)Übertragung selbst.

\section{Das vorliegende Fragment und Kriterien der Edition}

Das Maulbronner Fragment, das ursprünglich eine normale Foliantengröße aufgewiesen haben wird, ist zweispaltig geschrieben; durch die Verarbeitung als Buchinnendeckelschutz ist ein Textverlust von 4-5 Zeilen an der Oberkante und insbesondere im oberen Teil an den Außenrändern entstanden. Für die nachfolgende Edition sind die fehlenden Teile aus der mutmaßlich ältesten Handschrift (Paris, BnF, Ms. lat. 16.558) (= $P$ ) rekonstruiert. Für die Darstellung wurde entschieden, die eindeutigen Abkürzungen stillschweigend aufzulösen und bei Zeilenumbrüchen Bindestriche einzufügen, ansonsten aber die Schreibweise (auch hinsichtlich der Groß- und Kleinschreibung) und die Einzelzeilenform beizubehalten und die Interpunktion (gewöhnlich Hochpunkte) dem Kontext anzupassen (durch Kommata, Punkte und Doppelpunkte). In Fällen, in denen in einer Zeile erst der Beginn eines neuen Sinnabschnitts und dann der Übertrag der vorherigen Zeile steht, wurde die Zeile durch xb/xa aufgelöst; Korrekturen im Manuskript (=M) werden als solche dargestellt, ein Unterpunkt steht dabei für eine Streichung des Buchstabens. Die Bibelzitate sind in Fußnoten ausgewiesen; Nachweise der dem Lateinischen entsprechenden Talmudstellen werden am Rand abgebildet.

Bei dem Fragment handelt es sich inhaltlich um eine Aneinanderreihung von Exzerpten aus dem Talmudtraktat bSanhedrin (f. 22a-38b; sowohl Mischna als 
auch Gemara) ${ }^{29}$; diese Exzerpte stammen jedoch nicht, wie die Geschlossenheit der Vorlage vermuten lässt, aus der ersten umfangreichen Übertragung, sondern es handelt sich um eine Kopie aus der systematisierten dritten Übertragung bzw. Kompilation, hier aus der Zusammenstellung „De stulticiis“ (Über die Dummheiten). Die in dem vorliegenden Fragment gesammelten Exzerpte umfassen eine je teilweise Wiedergabe der Diskussion von mSanh. II, 5, einer Auslegung von Deuteronomium 17, 15 verknüpft mit Fragen der Haarpflege, der Scheidung von einer Frau bzw. des Verhaltens nach ihrem Tod, und dem Weintrinken, von mSanh. III, $3 \mathrm{zu}$ bestimmten Berufen, deren Zeugnis fragwürdig ist, mit einer stark verkürzten Zusammenfassung der nachfolgenden Diskussion, der Diskussionen zu mSanh. III, $5 \mathrm{zu}$ Verwandtschaftsverhältnissen und zu mSanh. III, 6-7 über die Paradiesschlange, zu mSanh. IV, 5 und dem Blut Abels, der Frage, ob Verbannung Sühne bewirke, sowie die Formung des ersten Adam. Die Bedeutung des Fragments liegt weniger in dem skizzierten Inhalt als vielmehr in seiner Existenz an sich, weil es den nunmehr achten bekannten Überlieferungsträger der Übertragungen der 1230er und 1240er Jahre darstellt.

\section{Ausblick}

Die lateinischen Übersetzungen wurden teilweise oder vollständig im 13. Jahrhundert kopiert; bislang sind acht Überlieferungsträger bekannt und teilweise oder vollständig erhalten. Wir haben jedoch fast keine zeitgenössischen Autoren, die auf den Talmud in der vorliegenden Übertragung zurückgegriffen haben, d.h. den Talmud gelesen und in eigenen Werken verwendet haben. Die einzigen Autoren, von denen wir das wissen, sind Albertus Magnus, der in seinem Matthäus-Kommentar an einer Stelle einmal auf diese Auszüge aus dem Talmud zurückkommt - es handelt sich aber um eine vereinzelte Nennung im äußerst umfangreichen Werk dieses vielleicht bedeutendsten Lehrers des Dominikanerordens! -, sowie der Verfasser der Pharetra von Theobaldus de Saxonia. Vielmehr - und das ist dann auch wieder ein Phänomen des Dominikanerordens - hat man den eigenen Übersetzungen nicht getraut, und in der Mitte des Jahrhunderts hat der Dominikaner Ramon Martí ein groß angelegtes Werk gegen Juden und Muslime geschrieben, den „Glaubensdolch" (Pugio fidei), und hat in diese Übertragung Neuübertragungen talmudischer Texte eingefügt; diese sind literarisch um einiges ansprechender ${ }^{30}$. Das heißt, der Dominikanerorden selbst beauftragt - wahrscheinlich sogar durch den

${ }^{29}$ Es handelt sich um Texte, die nicht im Breslauer Fragment, das ebenfalls eine Übersetzung aus dem Traktat bSanhedrin enthält, überliefert sind. Die Fragmente stammen, legt man paläografische Kriterien zugrunde, nicht aus der gleichen Handschrift.

30 Vgl. Raimundus Martini, Texte zur Gotteslehre. Pugio fidei I-III, 1-6. Lateinisch Hebräisch / Aramäisch - Deutsch, hg., übersetzt und eingeleitet von G.K. Hasselhoff, Freiburg u.a. 2014; weitere Bände sind geplant. 
gleichen Ordensgeneral - jemanden, um diese ersten Übertragungen zu ersetzen. Dennoch stellen sie ein wichtiges Zeugnis für die christliche Wahrnehmung der Transformation des mittelalterlichen Judentums dar. 


\section{Anhang: Edition}

fol. 1ra

\begin{tabular}{|c|c|c|}
\hline [1] & $\begin{array}{l}{ }^{1}[\text { Dicit rby Ysaac: In hora qua Salomon accepit } \\
\text { filiam Pharaonis in uxorem },{ }^{2} \text { Gabriel descendit }\end{array}$ & bSanh. 21b \\
\hline [2] & et fixit virgam in mari, et coincurvata est terra & \\
\hline [3] & circa eam, et super illam terram edificatum & \\
\hline 4 & fuit magnum castrum $\mathrm{R}$ ]ome. & \\
\hline 5 & [Alius non debet] equitare equ[um regis] & $\begin{array}{l}\text { bSanh. 22a } \\
(=\text { mSanh. II,5) }\end{array}$ \\
\hline 6 & [nec sedere in s]ede eius, nec ten[ere eius virg]am & \\
\hline 7 & $\begin{array}{l}\text { [glossa salomonis: id est] non debet concumbere cum muliere cum } \\
\text { qua rex }\end{array}$ & \\
\hline 8 & [concub]uit, nec ipsum uidere nudum nec quando radi & \\
\hline 9 & [tur n]ec in balneo, secundum quod scriptum est ${ }^{3}$ & \\
\hline 10 & [eum const] itues quem dominus deus tuus elegerit & \\
\hline 11 & [hoc est dictu]m timebis eum. Dicunt Raby & bSanh. 22a \\
\hline 12 & [Iacob et] raby Iohan: quia salomon bene & \\
\hline 13 & [potuit cont]rahere cum abysach, quod rex bene & \\
\hline 14 & [potest uti vir]ga regis, sed yadonias non potu- & \\
\hline $15 \mathrm{~b}$ & it. & \\
\hline $15 \mathrm{a}$ & [Dicit] Raby & bSanh. 22a \\
\hline 16 & [ele]azar: omnis qui relinquit pri & \\
\hline 17 & [ma]m uxorem, altare plorat super & \\
\hline 18 & [eum, unde] ${ }^{4}$ scriptum est ${ }^{5}$ operiebatis lacrimis & \\
\hline 19 & [altare] meum, fletu et gemitu quam ob & \\
\hline 20 & [causam qui]a dominus testificatus est inter te et ux & \\
\hline 21 & [orem] pubertatis. Tue. Quam tu despexisti. & \\
\hline 22 & {$[\mathrm{~V}]$ xor uiri non moritur, } & bSanh. 22a \\
\hline 23 & nisi petatur ab ea. census. et ipsa non & \\
\hline 24 & [habeat] unde restituat, secundum quod scriptum est: ${ }^{6}$ si & \\
\hline 25 & [n]on habes unde restituas. Quid est ut tol- & \\
\hline 26 & [1] as cubile tuum subtus te, id est uxorem. & \\
\hline 27 & Dicit Raby Iohan: Homo cuius prima & bSanh. 22a \\
\hline 28 & uxor moritur ita debet esse, quasi uideret & \\
\hline 29 & domum sanctuarij destrui coram se, sicut & \\
\hline
\end{tabular}

${ }^{1}$ P fol. 54 ra, lin. 23.

2 Cf. 1. Kön. 3, 1.

3 Dtn. 17, 15.

${ }^{4}$ P fol. 54rb.

5 Mal. 2, 13-14.

${ }^{6}$ Prvb. 22, 27.

Zeitschrift für Württembergische Landesgeschichte 74 (2015), S. 331-344.

(C) Kommission für geschichtliche Landeskunde in Baden-Württemberg und Württembergischer Geschichts- und Altertumsverein e.V.

ISSN 0044-3786 


\begin{tabular}{|c|c|c|}
\hline 30 & scriptum est ${ }^{7}$ fili ${ }^{8}$ hominis ecce ego tollo a & \\
\hline 31 & te desiderabilem oculorum tuorum et paulo & \\
\hline 32 & post $^{9}$ et mortua est uxor mea, uespere et iterum ${ }^{10}$ & \\
\hline 33 & Ecce ego polluam sanctuarium meum desi- & \\
\hline 34 & derabilem occulorum meorum. & \\
\hline 35 & In omnibus potest fieri restauracio nisi in prima & bSanh. 22a \\
\hline 36 & uxore, unde scriptum est: ${ }^{11}$ ut mulierem derelictam & \\
\hline 37 & uocauit te dominus, et uxorem ab ado- & \\
\hline 38 & lescencia abiectam. & \\
\hline
\end{tabular}

fol. 1rb

\begin{tabular}{|c|c|c|}
\hline$[1]$ & [Dicit rab Iuda: Nullus reperit suavitatem spiritus, nisi in prima & bSanh. 22a \\
\hline [2] & uxore, sicut scriptum est: $:^{12}$ letare cum muliere adolescencie tue. & \\
\hline$[3]$ & Dicit rab Samuel: mulier forma est & bSanh. 22b \\
\hline [4] & imperfecta ante quam cognoscatur, nec unquam & \\
\hline [5] & confirmabit amorem suum, nisi cum illo & \\
\hline 6 & qui facit] eam [vas, qui scilicet] & \\
\hline 7 & [impregnat vel] deflorat ea[m, unde] & \\
\hline 8 & scriptum est ${ }^{13}$ dominabitur tui [qui fecit te, & \\
\hline $9 \mathrm{~b}$ & vas scilicet.] & \\
\hline $9 \mathrm{a}$ & Quando uir & bSanh. 22b \\
\hline 10 & moritur non moritur nisi uxori su[e unde] & \\
\hline 11 & scriptum est: $:^{14}$ mortuus est elymelech ma- & \\
\hline 12 & ritus noemy nec uxor moritur nisi marito & \\
\hline 13 & unde scriptum est: ${ }^{15}$ Michi quando ueniebam de & \\
\hline 14 & mesopothamiam mortua est rachel. & \\
\hline 15 & Dicunt magistri: Rex debet & bSanh. 22b \\
\hline 16 & radi cottidie quia scriptum est: ${ }^{16}$ re- & \\
\hline 17 & gem in decore suo uidebunt oculi mei. & \\
\hline 18 & Magnus pontifex ab una ebdomada in aliam & \\
\hline 19 & ${ }^{17}$ quia cottidie innouantur in illa: sacerdos & \\
\hline
\end{tabular}

\footnotetext{
7 Ez. 24, 16.

${ }^{8}$ fil[i]j corr. $M$

9 Ez. 24, 18.

10 Ez. 24, 21.

${ }^{11}$ Jes. 54, 6 .

12 Prvb. 5, 18.

13 Jes. 54, 5.

14 Rut 1, 3.

15 Gen. 48, 7.

16 Jes. 33, 17.

17 P fol. $54 v a$.
}

Zeitschrift für Württembergische Landesgeschichte 74 (2015), S. 331-344.

(C) Kommission für geschichtliche Landeskunde in Baden-Württemberg und Württembergischer Geschichts- und Altertumsverein e.V.

ISSN 0044-3786 


\begin{tabular}{|l|l|l|}
\hline 20 & semel in .xxx. diebus quoniam scriptum est ${ }^{18} \mathrm{ca}^{-}$ & \\
\hline 21 & pud non radetur, nec comam nutrient. & \\
\hline 22 & Dicunt magistri: & bSanh. 22b \\
\hline 23 & sacerdotes huius temporis non debent bi- & \\
\hline 24 & bere uinum. Nescitur enim si domus sanctuarij fi[et.] & \\
\hline 26 & et tunc necesse esset habere sacerdotem unum & \\
\hline 27 & et si bibisset uinum non posset ministrasse & \\
\hline 28 & Immo posset enim parum dormire prius. hoc & \\
\hline 29 & enim dicit rame. uia unius mụ<i>liẹ $<$ a ris ${ }^{19}$ & \\
\hline 30 & Ita dicit raua: dum modo non biberit nisi unam & \\
\hline 31 & quartam uini, sed amplius quam quartam quan- & \\
\hline 32 & to plus dormiret, et tanto plus inebri- & \\
\hline $33 b$ & aretur. & bSanh. 22b $(=$ \\
\hline $33 a$ & Isti repelluntur & \\
\hline 34 & a testimonio. Lusor taxillorum et usurarius et qui 3$)$ \\
\hline 35 & habet columbas uolantes, et mercatores & \\
\hline 36 & septimi anni. ${ }^{20}$ Dicit Raby huza: hoc & bSanh. 25a-b \\
\hline 37 & intelligitur de illis qui aliud officium non exercent & \\
\hline 38 & Qui uendit terephot ${ }^{21}$ & \\
\hline
\end{tabular}

fol. 1va

\begin{tabular}{|l|l|l|}
\hline$[1]$ & [carnes animalis quod habet vitium circa pulverem vel huiusmodi, & bSanh. 25a-b \\
\hline$[2]$ & non admitatur ad testimonium. Qui habet columbas volantes & \\
\hline$[3]$ & et lusor, etiamsi non luderet nisi cum nucibus vel corticibus & \\
\hline$[4]$ & malogranatorum, repelluntur a testimonio. & \\
\hline$[5]$ & Qui dat ad usuram et qui accipit, uterque repellitur a testimonio. & \\
\hline 6 & Et quando erit eorum penitentia? Quando destruent] cartas & \\
\hline 7 & {$[$ suas et vere penitebunt] ita quod etiam goym } & \\
\hline 8 & {$[$ christianis, non dab]unt ad usuram. } & \\
\hline 9 & {$[$ Magis]tri addiderunt super illos: quos } & bSanh. 25b \\
\hline 10 & mysua 22 repellit a testimonio pastores cuius & \\
\hline 11 & cumque animalis et custodem curban, misericordia et elemosinas & \\
\hline 12 & et thelonarium, uiderunt enim quod illi ac- & \\
\hline 13 & cipiebant plus debito. & \\
\hline
\end{tabular}

\footnotetext{
18 Ez. 44, 20.

19 corr. $M]$ miliaris $P$

20 Cf. Lev. 25, 6.

21 terephot] terechot $P$

22 mysua sic] mysna $P$
}

Zeitschrift für Württembergische Landesgeschichte 74 (2015), S. 331-344.

(C) Kommission für geschichtliche Landeskunde in Baden-Württemberg und Württembergischer Geschichts- und Altertumsverein e.V.

ISSN 0044-3786 


\begin{tabular}{|c|c|c|}
\hline 14 & Dicit abaye: si quis commendatur de falso & bSanh. 26b-27a \\
\hline 15 & testimonio, testimonia que prius tule- & \\
\hline 16 & rat sunt falsa & \\
\hline 17 & Non morientẹs $<$ ur $>^{23}$ patres pro filijs, id est pre & bSanh. $27 \mathrm{~b}$ \\
\hline 18 & testamentum filiorum, nec filii pro patri- & \\
\hline 19 & bus, ${ }^{24}$ id est per testimonium patrum. & \\
\hline 20 & Unde habetis quod non est loquendum pro mo- & bSanh. 29a \\
\hline 21 & ta $^{25}$ tore $^{26}$. Dicit Raby Samuel: habemus & \\
\hline 22 & hoc per serpentem. Dicit enim Symeon: mul- & \\
\hline 23 & tas excusaciones habebat serpens, sed non & \\
\hline 24 & dixit eas et deus, quare non dixit illas quia & \\
\hline 25 & [n]on est loquendum pro incitatore. & \\
\hline 26 & In mysua ${ }^{27}$ dicitur ${ }^{28}$ uox sanguinis fratris tui: clamat & bSanh. 37b \\
\hline 27 & ad me de terra. Dicit Raby euehu: per & \\
\hline 28 & hoc postestis di<s $>\operatorname{cere}^{29}$, quod chayn fecit in abel & \\
\hline 29 & fratre suo plagas et plagas uulnera et & \\
\hline 30 & uulnera, quoniam nesciebat per quem locum ani- & \\
\hline 31 & ma debebat exire donec uenit ad collum. & \\
\hline 32 & Dicit· Raby huza: & bSanh. 37b \\
\hline 33 & a die .qua qua [die $]<$ terra $>^{30}$ aperuit os suum, & \\
\hline 34 & et suscepit sanguinem abel, non peraperuit & \\
\hline 35 & illud, unde scriptum est: ${ }^{31}$ a finibus terre $. \mathrm{fi}^{32} \mathrm{lau}-$ & \\
\hline 36 & des audiuimus, a finibus terre non ab ore & \\
\hline 37 & terre. Dicit ezechias: Nonne scriptum & \\
\hline 38 & est $^{33}$ et aperiens terra os suum deuorabit eos. & \\
\hline
\end{tabular}

fol. 1vb

\begin{tabular}{|l|l|l|}
\hline$[1]$ & $\begin{array}{l}\text { [Verum est quod pro malo aperuit postea os suum, sed non pro } \\
\text { bono. }\end{array}$ & \\
\hline$[2]$ & Dicit rab Iuda: captivitas remittit medietatem peccatorum, & bSanh. 37b \\
\hline$[3]$ & unde scriptum est de Cain: ${ }^{34}$ vagus et profugus eris & \\
\hline
\end{tabular}

23 corr. $M]$ morientur $P$

24 2. Kön. 14, 6.

${ }^{25} \mathrm{P}$ fol. $54 v b$.

${ }^{26}$ motatore $s i c$ ] incitatore $P$

27 mysua sic] mysna $P$

28 Gen. 4, 10.

29 corr. $M]$ discere $P$

30 corr. $M]$ a die qua terra $P$

31 Jes. 24, 16.

32 corr. $M$

33 Num. 16, 30; 26, 10.

${ }^{34}$ Gen. 4, 12. 


\begin{tabular}{|c|c|c|}
\hline 4 & super terram.Et paulo po]st: ${ }^{35}$ eggress[usque Cain] & \\
\hline 5 & [a facie Domini habitav]it profugus in $\mathrm{t}$ [erra non est ibi vagus]. & \\
\hline 6 & $\mathrm{D}$ [icit] Raby iuda & bSanh. 37b \\
\hline 7 & captiuitas condonat tria: g[ladium, fa- $]$ & \\
\hline 8 & mem et pestem secundum quod scriptum est: ${ }^{36}$ [qui habi-] & \\
\hline 9 & tauerit in urbe hac, fame mor[ietur et] & \\
\hline 10 & peste. Qui autem egressus fuerit [et transfugerit] & \\
\hline 11 & ad caldeos uivet. & \\
\hline 12 & Dicit Raby Johan: captiu[itas expur-] & bSanh. 37b \\
\hline 13 & gat omne peccatum. Vnde script[um est: ${ }^{37}$ scri-] & \\
\hline 14 & be uirum iustum sterilem etc. et al[ibi legitur: $\left.{ }^{38}\right]$ & \\
\hline 15 & filij iethome fuerunt asyi, s[alathihel, Mel-] & \\
\hline 16 & thyram, phaday etc. ergo multo[s habuit filios, ] & \\
\hline 17 & glossa salomonis: dicit quod hoc fuit propter [captivita] & \\
\hline 18 & tem. & \\
\hline 19 & Dicit Raby mehir: pulu[is ade pri] & bSanh. 38a-b \\
\hline 20 & mo fuit congregatus de toto [mundo] & \\
\hline 21 & unde ${ }^{39}$ scriptum est: ${ }^{40}$ imperfectum meu[m viderunt oculi] & \\
\hline 22 & tui, glossa salomonis: de omni quod oculi dei [videre] & \\
\hline 23 & possunt in terra fui ego creatus e[t hoc est $]$ & \\
\hline 24 & totus mundus, unde scriptum est: ${ }^{41}$ Isti sunt [oculi] & \\
\hline 25 & domini qui decurrunt in uniuersam terram. & \\
\hline 26 & Dicit Raua & bSanh. 38b \\
\hline 27 & corpus ade fuit factum de babylone, [glossa] & \\
\hline 28 & salomonis: propter hoc est babylon profunda, capud & \\
\hline 29 & de terra israel, qui altior et melior est. Aliis, & \\
\hline 30 & ita capud cetera membra fuerunt de alijs ter- & \\
\hline 31 & ris. Dicit Raby Iohan: Nates ipsius facti & \\
\hline 32 & sunt de akarad agma, glossa: platea est & \\
\hline 33 & [i]n babilone ualde profunda. & \\
\hline 34 & Dicit Raby aha: Duodecim hore sunt & bSanh. 38b \\
\hline 35 & diei. In prima fuit puluis ade congre- & \\
\hline 36 & gatus. In secunda fuit factus modulus, ad im- & \\
\hline 37 & primendum formam. In tercia fuerunt dis & \\
\hline 38 & tenta membra. In quarta infudit deus animam. & \\
\hline
\end{tabular}

35 Gen. 4, 16.

36 Jer. $21,9$.

37 Jer. 22, 30.

38 1. Chr. 3, 17-18.

39 P fol. 55 ra.

40 Ps. 139 [138V], 16.

${ }^{41}$ Za. 4, 10.

Zeitschrift für Württembergische Landesgeschichte 74 (2015), S. 331-344.

(C) Kommission für geschichtliche Landeskunde in Baden-Württemberg und Württembergischer Geschichts- und Altertumsverein e.V. 